\title{
Aptidão física relacionada ao desempenho motor em escolares de sete a 15 anos
}

CDD. 20.ed. 152.3

796.071

\author{
Samuel Carvalho DUMITH* \\ Virgílio Viana RAMIRES* \\ Matheus Jesuíno Alves SOUZA** \\ Daniel Souza MORAES ${ }^{* * * *}$ \\ Fabrício Godoy PETRY $Y^{* * * * *}$ \\ Eduardo Soldera OLIVEIRA ${ }^{* * * * *}$ \\ Sandro Viana RAMIRES \\ Alexandre Carriconde MARQUES
}

\begin{abstract}
Resumo
Os beneficios do desenvolvimento da aptidão fisica sobre a saúde estão bem evidenciados na literatura científica. 0 objetivo do presente estudo foi descrever a aptidão fisica relacionada ao desempenho motor de crianças e adolescentes e examinar as diferenças de acordo com sexo, idade, tipo de escola (pública ou privada) e região geográfica da escola (urbana ou rural). Uma bateria de cinco testes motores foi aplicada a 526 alunos entre sete e 15 anos do ensino fundamental de Rio Grande, RS: salto em distância parado, arremesso de "medicineball", barra modificada, corrida de 20 metros e quadrado. Os resultados apontam que o desempenho em todos os testes foi superior para os rapazes e aumentou diretamente com a faixa etária. Estes foram os principais preditores da aptidão física dentre as variáveis examinadas. 0 tipo de escola só teve influência sobre o desempenho no teste de arremesso de "medicine-ball", em que alunos da rede privada obtiveram valores médios maiores. Escolares da zona urbana atingiram um melhor desempenho em todos os testes, exceto no teste de barra modificada (sem diferença por região geográfica). A realização de pesquisas com o mesmo enfoque em outras regiões do Brasil permitirá explorar a consistência destes achados e compreender possiveis diferenças.
\end{abstract}

UnIteRMos: Atividade motora; Exercício; Desempenho atlético; Crianças; Adolescentes.

\section{Introdução}

Os benefícios do desenvolvimento da aptidão física sobre a saúde estão bem evidenciados na literatura científica. Dentre eles, destacam-se: menor a incidência dos fatores de risco para doenças crônicas, redução da adiposidade total e abdominal, melhora da saúde mental e corporal e aumento do desempenho acadêmico (Glaner, 2003; KvaAvik, Klepp, Tell, Meyer \& Batty, 2009; Ortega, Ruiz, Castillo \& Sjostrom, 2008). Além disso, a aptidão física está mais fortemente relacionada à prevenção da síndrome metabólica do que a atividade física (Rizzo, Ruz, Hurtig-Wennlof, Ortega \& SJostrom, 2007).

No entanto, há vários estudos mostrando que os níveis de aptidão física têm declinado ao longo das últimas décadas (MALINA, 1996; TOMKINSON \& OldS,
2007; Tomkinson, Olds, Kang \& Kim, 2007; WesterSTAHL, BarNeKow-BerGKVIST, HedBERG \& JansSON, 2003). Estimativas provenientes dos Estados Unidos apontam que a prevalência de aplicação de testes de aptidão física no ano 2000 foi de $65 \%$ em escolas de todos os níveis (Morrow Junior, Fulton, Brener \& KoHL, 2008). No Brasil, não há dados científicos acerca dessa proporção, mas percebe-se que esta prática está em decadência no contexto escolar (observação empírica).

Conforme GuEdes (2007), a aptidão física pode ser dividida em dois componentes. O primeiro corresponde à aptidão física relacionada à saúde e envolve basicamente as seguintes capacidades físicas: resistência cardiorrespiratória, força/resistência muscular e flexibilidade (GUEDES, 2007). O segundo componente

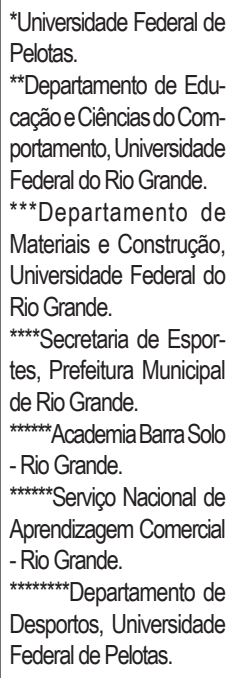

*Universidade Federal de Pelotas.

**Departamento de Educaçãoe Ciências do Comportamento, Universidade Federal do Rio Grande. ***Departamento de Materiais e Construção, Universidade Federal do Rio Grande.

${ }^{* * * *}$ Secretaria de Esportes, Prefeitura Municipal de Rio Grande.

**k***AcademiaBarraSolo - Rio Grande.

******Serviço Nacional de Aprendizagem Comercial - Rio Grande.

********Departamento de Desportos, Universidade Federal de Pelotas. 
diz respeito à aptidão física relacionada ao desempenho motor, eabrange as seguintes habilidades: potência (ou força explosiva), velocidade, agilidade, coordenação e equilíbrio (GUEDES, 2007). A maior parte dos estudos encontrados na literatura avaliou apenas o primeiro componente. Poucos estudos sobre o segundo foram localizados. Ainda assim, a maioria deles é apenas descritivo.

Um estudo indicou que o nível de esforço físico nas aulas deEducação Física não ésuficiente para aumentar a aptidão física (GUEDES \& GUEDES, 2001). Outro estudo nacional feito com escolares identificou que a prática de atividades física com intensidade moderada a vigorosa explicou somente $4-8 \%$ da variabilidade do consumo máximo de oxigênio (Guedes, Guedes, Barbosa \& Oliveira, 2002).

\section{Método}

\section{Delineamento}

Trata-se de um estudo epidemiológico com desenho transversal. Esta pesquisa foi desenvolvida como parte de um projeto nacional, denominado "Projeto Esporte Brasil" (PROESP). Este projeto foi lançado em 2002 pelo Ministério dos Esportes, e teve, dentre outros objetivos, traçar o perfil de aptidão física das crianças e adolescentes brasileiros. Detalhes adicionais sobre o respectivo projeto podem ser conferidos em outra publicação (MARQues, Gaya, Silva \& Torres, 2005), bem como no seguinte endereço eletrônico: www.proesp.ufrgs.br.

\section{Aspectos éticos}

O protocolo do estudo foi aprovado pelo Comitê de Ética em Pesquisa da Universidade Federal de Pelotas. Um consentimento verbal foi obtido dos diretores de cada instituição de ensino incluída na amostra. Além disso, os pais ou responsáveis por cada aluno deveriam assinar um termo de consentimento autorizando-o a participar do estudo.

\section{Amostra e amostragem}

O presente estudo ocorreu no município de Rio Grande, RS, cuja população estimada é de aproximadamente 200 mil pessoas. A amostra foi selecionada em dois estágios. Primeiro, selecionaramse 10 escolas com ensino fundamental (de 90, na época de estudo), as quais foram previamente estratificadas por tipo de escola (pública e privada)
Acrescentando, a aptidão física adquirida na infância e adolescência tende a perdurar até a fase adulta (MALINA, 1996; TwISK, KEMPER \& VAN MECHELEN, 2000).

Logo, a mensuração da aptidão física em jovens consiste em uma importante ferramenta disponível aos professores de Educação Física para avaliar e monitorar o desempenho dos seus alunos. Além disso, é importante determinar se o nível de aptidão física difere de acordo com determinadas características, tanto do aluno, quanto do contexto. Dessa forma, o objetivo do presente estudo foi descrever a aptidão física relacionada ao desempenho motor de crianças e adolescentes e examinar as diferenças de acordo com sexo, idade, tipo de escola e região geográfica da escola.

e pela localidade geográfica (rural e urbana). Com o intuito de assegurar a proporcionalidade existente no município, foram selecionadas oito escolas públicas e duas privadas, bem como três rurais e sete urbanas.

O próximo passo do processo amostral consistiu na seleção das turmas de cada série. Quando a escola possuísse apenas uma turma, esta era automaticamente selecionada. Se tivesse mais de uma turma por série, era feita uma seleção aleatória, com probabilidade proporcional ao número de escolares em cada série no município de Rio Grande.

Foram selecionados, em média, aproximadamente 70 escolares por série do ensino fundamental ( $1^{\mathrm{a}} \mathrm{a}$ $8^{a}$ ) de cada escola incluída na amostra. Os critérios de inclusão eram: estar devidamente matriculado na escola no respectivo ano letivo; e ter idade entre sete e 15 anos. Aqueles escolares cuja idade não atingisse ou extrapolasse a faixa etária do estudo, foram avaliados junto com seus colegas de classe, porém foram posteriormente excluídos das análises. Os alunos que não quiseram participar, tinham alguma limitação física para fazer os testes, que não trouxeram o termo de consentimento assinado ou que se ausentaram no dia das avaliações, foram considerados como perdas. A reposição das perdas não foi permitida para não causar um possível viés de seleção.

\section{Logística}

A equipe de examinadores foi formada por seis profissionais de Educação Física, todos devidamente treinados e padronizados para realizar os testes e medidas do estudo. Um estudo piloto, envolvendo 
cerca de 100 alunos de quatro turmas de uma escola não incluída na amostra, foi realizado com o propósito de testar os instrumentos e a logística do estudo. Ao longo do trabalho de campo, os alunos eram informados com pelo menos um dia de antecedência sobre a pesquisa e os procedimentos necessários para participar das avaliações físicas. Os testes eram feitos preferencialmente no período das aulas de Educação Física. A coleta de dados ocorreu entre os meses de setembro e novembro de 2004.

\section{Variáveis dependentes}

As variáveis dependentes corresponderam aos resultados dos testes de aptidão física relacionada ao desempenho motor. Salienta-se que os testes de aptidão física relacionada à saúde também foram aplicados, porém foram objeto de pesquisa de outro estudo já publicado (Dumith, Azevedo \& Rombaldi, 2008). Desse modo, cinco testes foram empregados neste trabalho, os quais estão descritos resumidamente a seguir (entre parênteses, está a principal habilidade motora envolvida em cada um deles).

\section{Salto em distância parado (potência muscular de membros inferiores)}

O aluno ficava parado, com os pés atrás de uma linha feita no chão. Por um movimento de flexão do joelho e quadril deveria pegar impulsão e saltar a distância máxima que conseguisse. Uma tentativa era permitida antes do salto oficial. A distância entre a linha no chão e a parte posterior dos pés era mensurada em centímetros por meio de uma fita métrica.

\section{Arremesso de "medicine-ball"}

(potência muscular de membros superiores)

O aluno devia sentar no chão, com as costas encostadas na parede, e o quadril num ângulo de $90^{\circ}$. Com as duas mãos, devia segurar, encostado no peito, uma bola pesando $1,5 \mathrm{~kg}$. Ao sinal, jogavam a bola para frente, numa trajetória parabólica, de modo a lançarem o mais longe que conseguisse. A distância da parede até o primeiro ponto onde a bola tocasse o solo era registrada em centímetros, por meio de uma fita métrica.

Barra modificada (força de membros superiores)

Uma armação de madeira foi construída de modo a posicionar a barra em diversas alturas. $\mathrm{O}$ aluno devia deitar sobre um colchonete, posicionado sob a barra.
A barra era colocada numa altura em que os braços do aluno ficassem totalmente estendidos para cima. $\mathrm{O}$ movimento consistia numa flexão dos cotovelos, com o quadril totalmente reto, até o seu queixo atingir a altura da barra. $\mathrm{O}$ aluno devia realizar o máximo de repetiçôes que conseguisse, sem tocar o tronco ou o quadril no solo. Eram contabilizados apenas os movimentos corretos, e não havia limite de tempo.

\section{Corrida de 20 metros (velocidade)}

Uma pista de 20 metros, reta e sem obstáculos, era demarcada, preferencialmente na quadra de esportes da escola. O aluno deveria percorrer a distância da pista no menor tempo que conseguisse. O tempo era registrado por um cronômetro em segundos e centésimos. O teste era feito por um aluno de cada vez. Salienta-se que quanto maior o tempo, pior o desempenho.

\section{Quadrado (agilidade e coordenação)}

Quatro cones, separados por uma distância de quatro metros entre si, eram posicionados no chão de modo a formar a figura de um quadrado. Eram feitas quatro corridas entre os cones (descritas abaixo), alternando o sentido dos movimentos, sendo que o aluno deveria tocar com a mão sobre o cone quando o atingisse. Os alunos partiam de trás do primeiro cone até o que estivesse em sua diagonal (1), depois iam em direção ao cone situado a sua esquerda (2), depois novamente em diagonal corriam em direção ao terceiro cone (3), e por fim retornavam à direita ao cone de onde partiram (4). Cada aluno era estimulado a memorizar a sequência de movimentos e realizá-la no menor tempo possível. O tempo era registrado em segundo e centésimos por meio de um cronômetro. Salienta-se que quanto maior o tempo, pior o desempenho.

\section{Variáveis independentes}

Foram consideradas como variáveis independentes e possíveis fatores de confusão: sexo (masculino ou feminino); idade em anos completos e posteriormente categorizada em três grupos (sete a nove, 10 a 12 e 13 a 15 anos); tipo de escola (pública ou privada); e localidade geográfica da escola (rural ou urbana).

\section{Procedimentos estatísticos}

Para a descrição dos testes empregados foram utilizadas as medidas da estatística descritiva para variáveis 
contínuas. A descrição das variáveis independentes se deu por meio de proporções. As análises cruzando os escores de cada teste com as variáveis independentes foram feitas por ANOVA (análise de variância). As análises multivariáveis foram feitas empregando-se a técnica de regressão linear múltipla, ajustando-se o efeito de cada variável independente para as demais. $\mathrm{O}$ nível de significância estatística foi de 5\% para testes bicaudais. A limpeza do banco e análises dos dados foi feita no programa STATA (STATACORP, TX, 2000).

\section{Resultados}

Ao todo, foram avaliados 526 escolares entre sete e 15 anos do ensino fundamental de Rio Grande. Destes, $52,7 \%$ pertenciam ao sexo masculino, $12,9 \%$ estudavam em escolas particulares e $90,7 \%$ estudavam em escolas da zona urbana do município. A média de idade foi 11,0 anos (DP = 2,1). A descrição sumária dos valores obtidos em cada teste relacionado ao desempenho motor está apresentada na TABELA 1.

TABELA 1 - Média, desvio-padrão(DP), mediana, percentis 25 (P25) e 75 (P75) e valores mínimos (min) e máximos (max) das variáveis relacionadas ao desempenho motor.

\begin{tabular}{lccccc}
\hline \multicolumn{1}{c}{ Teste } & Média & DP & Mediana & P25 - P75 & Min.- Máx. \\
\hline Salto em distância (cm) & 130,9 & 29,8 & 128 & $110-148$ & $67-236$ \\
Arremesso de "medicine-ball” (m) & 2,41 & 0,82 & 2,25 & $1,8-2,8$ & $1,1-5,6$ \\
Barra modificada (repetiçóes) & 3,6 & 4,4 & 2 & $0-6$ & $0-29$ \\
Corrida de 20 metros (segundos) & 4,15 & 0,52 & 4,13 & $3,81-4,46$ & $2,59-5,69$ \\
Quadrado (segundos) & 6,94 & 0,77 & 6,87 & $6,34-7,43$ & $5,18-9,37$ \\
\hline
\end{tabular}

Os resultados das análises brutas e ajustadas para cada um dos cinco testes aplicados estão apresentados nas TABELAS 2 a 6. Quanto ao desempenho no teste de salto em distância, observa-se que foi superior para os rapazes, para os escolares da zona urbana, e que aumentou com a idade (TABELA 2). Não houve diferença conforme o tipo de escola (pública ou privada).

TABELA 2 - Análise bruta e ajustada do desempenho no teste de salto em distância (valores expressos em centímetros).

${ }^{*}$ Ajuste para as demais variáveis incluídas na tabela.

\begin{tabular}{|c|c|c|c|c|c|c|}
\hline \multirow{2}{*}{ Variável } & \multicolumn{3}{|c|}{ Análise bruta } & \multicolumn{3}{|c|}{ Análise ajustada* } \\
\hline & Média & DP & Valor $\mathbf{p}$ & Média & IC95\% & Valor $\mathbf{p}$ \\
\hline \multicolumn{7}{|l|}{ Sexo } \\
\hline Masculino & 142,4 & 30,4 & \multirow{2}{*}{$<0,001$} & 140,8 & $138,1-143,6$ & \multirow{2}{*}{$<0,001$} \\
\hline Feminino & 118,0 & 23,2 & & 119,7 & $116,8-122,7$ & \\
\hline \multicolumn{7}{|l|}{ Idade (anos) } \\
\hline $7-9$ & 110,8 & 18,6 & \multirow{3}{*}{$<0,001$} & 113,3 & $109,8-116,7$ & \multirow{3}{*}{$<0,001$} \\
\hline $10-12$ & 131,1 & 24,1 & & 131,3 & $129,2-133,3$ & \\
\hline $13-15$ & 152,1 & 34,1 & & 149,3 & $145,7-152,7$ & \\
\hline \multicolumn{7}{|l|}{ Tipo de escola } \\
\hline Pública & 130,7 & 30,4 & \multirow{2}{*}{0,70} & 130,7 & $128,6-132,9$ & \multirow{2}{*}{0,79} \\
\hline Privada & 132,2 & 25,4 & & 131,6 & $126,0-137,2$ & \\
\hline \multicolumn{7}{|l|}{ Zona geográfica } \\
\hline Urbana & 132,5 & 30,0 & \multirow[t]{2}{*}{$<0,001$} & 131,8 & $129,6-133,9$ & \multirow{2}{*}{0,007} \\
\hline Rural & 114,8 & 21,4 & & 122,0 & $115,2-128,7$ & \\
\hline
\end{tabular}


O desempenho no teste de arremesso de "medicine-ball" foi significativamente superior para os rapazes, para os escolares da rede privada e para os que estudavam em escolas da zona urbana (TABELA 3). Além disso, observa-se que aumentou de acordo com as categorias de faixa etária.

TABELA 3 - Análise bruta e ajustada do desempenho no teste de arremesso de "medicine-ball” (valores expressos em metros).

\begin{tabular}{|c|c|c|c|c|c|c|c|}
\hline \multirow{2}{*}{ Variável } & \multicolumn{3}{|c|}{ Análise bruta } & \multicolumn{3}{|c|}{ Análise ajustada* } & \multirow{3}{*}{$\begin{array}{l}\text { *Ajuste para as demais } \\
\text { variáveis incluídas na } \\
\text { tabela. }\end{array}$} \\
\hline & Média & DP & Valor $\mathbf{p}$ & Média & IC95\% & Valor $\mathbf{p}$ & \\
\hline \multicolumn{7}{|c|}{ tabela. } & \\
\hline Masculino & 2,68 & 0,93 & \multirow{2}{*}{$<0,001$} & 2,62 & $2,56-2,68$ & \multirow{2}{*}{$<0,001$} & \\
\hline Feminino & 2,11 & 0,55 & & 2,18 & $2,11-2,24$ & & \\
\hline
\end{tabular}

Idade (anos)

$\begin{array}{rrrrrrr}7-9 & 1,72 & 0,34 & & 1,73 & 1,66-1,81 & \\ 10-12 & 2,36 & 0,55 \\ 13-15 & 3,25 & 0,87 & & <, 001 & 2,42 & 2,38-2,47\end{array}<0,001$

Tipo de escola

$\begin{array}{lllllll}\text { Pública } & 2,36 & 0,79 & 0,001 & 2,37 & 2,32-2,42 & <0,001 \\ \text { Privada } & 2,73 & 0,96 & & 2,71 & 2,58-2,84 & \end{array}$

Zona geográfica

$\begin{array}{rrrrrr}\text { Urbana } & 2,48 & 0,82 & <0,001 & 2,45 & 2,40-2,50 \\ \text { Rural } & 1,69 & 0,33 & & 2,03 & 1,87-2,18\end{array}<0,001$

Com relação ao desempenho no teste de barra modificado, observa-se que foi maior para os rapazes e para aqueles de maiores faixas etárias (TABELA
4). No entanto, não houve diferenças significativas de acordo com o tipo de escola nem com a região geográfica.

TABELA 4 - Análise bruta e ajustada do desempenho no teste de barra modificada (número de repetições).

\begin{tabular}{|c|c|c|c|c|c|c|c|c|}
\hline \multirow{2}{*}{\multicolumn{2}{|c|}{ Variável }} & \multicolumn{3}{|c|}{ Análise bruta } & \multicolumn{3}{|c|}{ Análise ajustada* } & \multirow{3}{*}{$\begin{array}{l}\text { *Ajuste para as demais } \\
\text { variáveis incluídas na } \\
\text { tabela. }\end{array}$} \\
\hline & & Média & DP & Valor $\mathbf{p}$ & Média & IC95\% & Valor $\mathbf{p}$ & \\
\hline \multicolumn{8}{|c|}{ tabela. } & \\
\hline & Masculino & 5,3 & 4,9 & \multirow{2}{*}{$<0,001$} & 5,2 & $4,7-5,7$ & \multirow{2}{*}{$<0,001$} & \\
\hline & Feminino & 1,7 & 2,6 & & 1,8 & $1,3-2,3$ & & \\
\hline
\end{tabular}

Idade (anos)

$\begin{array}{rrrrrrr}7-9 & 2,4 & 3,2 & & 2,4 & 1,8-3,0 & \\ 10-12 & 3,2 & 3,6 & <0,001 & 3,6 & 3,3-4,0 & <0,001 \\ 13-15 & 5,6 & 5,9 & & 4,9 & 4,3-5,4 & \end{array}$

Tipo de escola

$\begin{array}{lllllll}\text { Pública } & 3,7 & 4,4 & & 3,7 & 3,3-4,0 & 0,20 \\ \text { Privada } & 3,3 & 3,9 & 0,55 & 3,0 & 2,0-4,0 & \end{array}$

Zona geográfica

\begin{tabular}{rrrrrrr} 
Urbana & 3,7 & 4,8 & 0,07 & 3,7 & $3,3-4,0$ & 0,21 \\
Rural & 2,5 & 3,0 & & 2,9 & $1,7-4,0$ & 0,0 \\
\hline
\end{tabular}


No que se refere ao teste de corrida de 20 de maiores faixas etárias (TABELA 5). Não metros, o desempenho foi maior para os rapazes, houve diferença entre escolares da rede pública para os escolares da zona urbana e para aqueles e privada.

TABELA 5 -Análise bruta e ajustada do desempenho no teste de corrida de 20 metros (valores expressos em segundos).

*Ajuste para as demais variáveis incluídas na tabela.

\begin{tabular}{|c|c|c|c|c|c|c|}
\hline \multirow{2}{*}{ Variável } & \multicolumn{3}{|c|}{ Análise bruta } & \multicolumn{3}{|c|}{ Análise ajustada* } \\
\hline & Média & DP & Valor $\mathbf{p}$ & Média & IC95\% & Valor $\mathbf{p}$ \\
\hline \multicolumn{7}{|l|}{ Sexo } \\
\hline Masculino & 3,95 & 0,50 & \multirow{2}{*}{$<0,001$} & 3,98 & $3,93-4,02$ & \multirow{2}{*}{$<0,001$} \\
\hline Feminino & 4,37 & 0,44 & & 4,35 & $4,30-4,40$ & \\
\hline \multicolumn{7}{|l|}{ Idade (anos) } \\
\hline $7-9$ & 4,53 & 0,44 & \multirow{3}{*}{$<0,001$} & 4,45 & $4,39-4,51$ & \multirow{3}{*}{$<0,001$} \\
\hline $10-12$ & 4,10 & 0,41 & & 4,14 & $4,11-4,18$ & \\
\hline $13-15$ & 3,83 & 0,52 & & 3,84 & $3,78-3,90$ & \\
\hline \multicolumn{7}{|l|}{ Tipo de escola } \\
\hline Pública & 4,16 & 0,51 & \multirow{2}{*}{0,59} & 4,15 & $4,12-4,19$ & \multirow{2}{*}{0,73} \\
\hline Privada & 4,12 & 0,55 & & 4,14 & $4,04-4,23$ & \\
\hline \multicolumn{7}{|l|}{ Zona geográfica } \\
\hline Urbana & 4,12 & 0,51 & \multirow{2}{*}{$<0,001$} & 4,13 & $4,09-4,17$ & \multirow{2}{*}{$<0,001$} \\
\hline Rural & 4,48 & 0,46 & & 4,36 & $4,24-4,48$ & \\
\hline
\end{tabular}

Por último, observa-se que o desempenho no teste do quadrado, assim como no teste acima mencionado, foi superior para os rapazes, para os escolares da zona urbana e para aqueles de maiores faixas etárias
(TABELA 6). No entanto, após ajustes para as demais variáveis independentes, os alunos de escolas públicas obtiveram um melhor desempenho, apesar da pequena diferença em relação àqueles de escolas particulares.
*Ajuste para as demais variáveis incluídas na tabela.
TABELA 6 - Análise bruta e ajustada do desempenho no teste do quadrado (valores expressos em segundos).

\begin{tabular}{|c|c|c|c|c|c|c|}
\hline \multirow{2}{*}{ Variável } & \multicolumn{3}{|c|}{ Análise bruta } & \multicolumn{3}{|c|}{ Análise ajustada* } \\
\hline & Média & DP & Valor $\mathbf{p}$ & Média & IC95\% & Valor $\mathbf{p}$ \\
\hline \multicolumn{7}{|l|}{ Sexo } \\
\hline Masculino & 6,61 & 0,69 & \multirow{2}{*}{$<0,001$} & 6,64 & $6,57-6,71$ & \multirow{2}{*}{$<0,001$} \\
\hline Feminino & 7,31 & 0,69 & & 7,28 & $7,20-7,35$ & \\
\hline \multicolumn{7}{|l|}{ Idade (anos) } \\
\hline $7-9$ & 7,52 & 0,62 & \multirow{3}{*}{$<0,001$} & 7,34 & $7,26-7,43$ & \multirow{3}{*}{$<0,001$} \\
\hline $10-12$ & 6,84 & 0,68 & & 6,93 & $6,88-6,98$ & \\
\hline $13-15$ & 6,51 & 0,73 & & 6,52 & $6,44-6,61$ & \\
\hline \multicolumn{7}{|l|}{ Tipo de escola } \\
\hline Pública & 6,93 & 0,76 & \multirow{2}{*}{0,21} & 6,91 & $6,87-6,97$ & \multirow{2}{*}{0,02} \\
\hline Privada & 7,05 & 0,82 & & 7,10 & $6,96-7,24$ & \\
\hline \multicolumn{7}{|l|}{ Zona geográfica } \\
\hline Urbana & 6,88 & 0,75 & \multirow{2}{*}{$<0,001$} & 6,89 & $6,84-6,94$ & \multirow{2}{*}{$<0,001$} \\
\hline Rural & 7,59 & 0,74 & & 7,45 & $7,29-7,62$ & \\
\hline
\end{tabular}


Os modelos de regressão linear múltipla apontaram que as variáveis sexo, idade, tipo de escola e localidade geográfica da escola explicaram, conjuntamente, $40 \%, 64 \%, 22 \%, 41 \%$ e $44 \%$ da variabilidade dos testes de salto em distância, arremesso de "medicineball", barra modificada, corrida de 20 metros e quadrado, respectivamente. $\mathrm{O}$ principal preditor isolado de cada um destes testes, com exceção da barra, foi a idade (em anos), seguido pelo sexo (este foi o principal preditor no teste da barra). Localidade geográfica e tipo de escola explicaram apenas uma pequena parcela da variância de cada teste.

\section{Discussão}

O objetivo deste estudo foi descrever os componentes da aptidão relacionada ao desempenho motor em jovens de um município localizado ao Sul do Brasil, bem como examinar se há diferença conforme sexo, idade, tipo de escola e região geográfica da escola. Uma bateria de cinco testes motores foi aplicada a 526 alunos entre sete e 15 anos do ensino fundamental.

Os resultados apontam que o desempenho em todos os testes foi superior para os rapazes e aumentou diretamente com a faixa etária. Aliás, estes foram os principais preditores da aptidão física dentre as variáveis examinadas, sugerindo que as características de cunho biológico respondem pela maior parte da variabilidade dos níveis de aptidão física, ao menos para crianças e adolescentes. $\mathrm{O}$ desempenho motor crescente com a idade e maior para o sexo masculino é um achado consistente na literatura, também sendo verificado, por exemplo, em um estudo feito com 1800 escolares de sete a 17 anos de Londrina, PR (GuedEs, 2007),

O tipo de escola só teve influência sobre o desempenho no teste de arremesso de "medicine-ball", em que os escolares da rede privada atingiram escores médios superiores aos da rede pública. Ausência de diferença pelo tipo de escola, nesta mesma população, também foi notada para os componentes de aptidão física relacionadas à saúde (Dumith, AzEvedo \& ROMBALDI, 2008). Quando levado em consideração o nível de atividade física, dois estudos nacionais também não notaram diferenças para alunos de escolas públicas e privadas (Hallal, Matsudo, Matsudo, Araújo, Andrade \& Bertoldi, 2005; Silva, Rivera, Ferraz, Pinheiro, Alves, Moura \& Carvalho, 2005). Esses achados sugerem não haver diferença na qualidade das aulas de Educação Física entre escolas da rede pública ou privada. Outra hipótese é que, havendo diferenças, as aulas de Educação Física não exercem efeito sobre o nível de aptidão física dos indivíduos. No entanto, tais hipóteses precisam ser mais bem exploradas por outros estudos delineados especificamente com este objetivo.
Escolares da zona urbana atingiram um melhor desempenho em todos os testes, exceto no teste de barra modificada. Neste, o número de repetições até foi maior do que os escolares da zona rural, mas não atingiu significância estatística, provavelmente devido à sua grande variabilidade amostral, o que pode ter afetado o poder das análises. Um estudo com escolares do norte gaúcho e oeste catarinense constatou que os rapazes da zona rural possuem aptidão física superior aos da zona urbana (GLANER, 2005). No entanto, no trabalho citado, foram analisados apenas os componentes de aptidão física relacionados à saúde. Por sua vez, outro estudo desenvolvido com escolares de sete a 10 anos do sexo masculino de Portugal encontrou resultados contraditórios, isto é, rapazes da zona urbana atingiram níveis superiores em alguns testes e inferiores em outros (Rodrigues, Bezerra \& Saraiva, 2005). Dessa forma, o efeito da localização geográfica sobre o nível de aptidão física ainda não está bem determinado, e pode variar conforme o contexto da população estudada. Além disso, tal efeito pode ser influenciado pelo estado socioeconômico, bem como por outros fatores, tanto ambientais (por exemplo, disponibilidade de espaços públicos para a prática de esportes) quanto sociais (por exemplo, influência dos amigos e pessoas próximas no comportamento relacionado à prática de atividade física).

As variáveis examinadas (sexo, idade, tipo de escola e região geográfica) explicaram cerca de dois terços $(65 \%)$ da variância no teste de arremesso de "medicine-ball". Para os testes de salto em distância, corrida de 20 metros e quadrado, tais valores ficaram próximos a 50\%. Entretanto, quando considerado o desempenho no teste de barra, essas variáveis explicaram apenas cerca de $20 \%$ de sua variabilidade. Uma possível explicação consiste na distribuição assimétrica (assimetria à direita) observada para este teste, diferentemente dos demais que apresentaram distribuição próxima da normalidade. Salienta-se, contudo que, mesmo se fosse utilizado um teste não-paramétrico (Kruskall-Wallis, por exemplo) 
os resultados permaneceriam basicamente os mesmos daqueles apresentados nas tabelas. Logo, optou-se pela utilização de testes paramétricos e pela não-transformação das variáveis para facilitar a compreensão dos achados.

Vale mencionar que um ajuste adicional para o índice de massa corporal (IMC) também foi feito, com o intuito de verificar se o desempenho em cada teste pudesse estar sendo confundido pelo IMC. No entanto, os resultados ficaram muito semelhantes e, portanto, não foram incluídos neste artigo. Isso significa que os achados apresentados neste estudo são independentes do peso e altura de cada indivíduo.

Ressalta-se que o desempenho avaliado refere-se apenas ao escore bruto obtido em cada teste, não tendo sido utilizado nenhum padrão de referência para classificar tais valores. Essa escolha se deu pela diversidade de critérios existentes na literatura e pelo fato de que esses valores normativos mudam de acordo com a população. Dessa forma, optou-se por descrever os valores absolutos de cada teste e comparálos segundo algumas características.

Algumas vantagens do estudo merecem ser destacadas. Primeiro, o método de seleção amostral permitiu que a amostra de jovens selecionados fosse representativa dos escolares do ensino fundamental de Rio Grande, RS. Segundo, a realização dos testes feita geralmente pelo mesmo avaliador (cada examinador ficou responsável pela aplicação de um a dois testes) atenuou a possibilidade de viés de mensuração. Terceiro, foram aplicados testes bem conhecidos e utilizados em várias baterias de aptidão física (AAHPERD, 1976; COMMITTEE FOR THE Development of Sport, 1988; Guedes \& Guedes, 2002; WelK, Marrow \& Falls, 2002).
Dentre as limitaçôes do estudo, estão a não-descrição das perdas, uma vez que estas não foram registradas. No entanto, a equipe que trabalhou na coleta de dados presenciou muito poucas recusas de alunos. A maior parte dos que não puderam participar das avaliaçōes foi porque não trouxeram o termo de consentimento assinado pelos pais ou responsáveis (embora muitos desses demonstrassem interesse em participar). A ausência de uma variável de maturação sexual/biológica também deve ser salientada, uma vez que ela difere entre e dentro de cada gênero e pode influenciar o nível de aptidão física. Outra limitação consiste na ausência da mensuração de um indicador socioeconômico do próprio aluno e de outras variáveis como cor da pele, por exemplo. Cabe ressaltar, contudo, que a prioridade estava na bateria de testes motores e logisticamente a coleta de outras variáveis, mesmo que por meio de questionário, era um tanto complicada logisticamente.

Como recomendações, sugere-se a realização de pesquisas com o mesmo enfoque em outras regiōes do Brasil, a fim de testar a consistência dos achados e procurar entender possíveis diferenças. Também se propõem que testes de aptidão física sejam administrados com maior frequência dentro do ambiente escolar, e que os alunos tenham seu desempenho monitorado ao longo do ano letivo pelos professores de Educação Física. Atenção especial deve ser dada aos escolares da zona rural, pois apresentaram desempenho inferior aos seus pares de escolas da zona urbana. No entanto, os resultados obtidos por alunos de escolas públicas e privadas foram muito semelhantes para os testes considerados. O desenvolvimento da aptidão física na infância e na adolescência deve ser estimulado como um meio de melhorar a condição de saúde tanto nesta fase da vida quanto na idade adulta.

\section{Abstract}

Physical fitness related to motor performance in scholars aged seven to 15 years

There is strong evidence regarding the benefits of physical fitness development upon health. The aim of this study was to describe the physical fitness related to motor performance in children and adolescents, and to examine if there are differences according to sex, age, type of school (public or private) and geographic region (urban or rural). A battery comprising of five motor tests were administered to 526 students aged seven to 15 years from elementary schools of Rio Grande, Brazil: stationary long jump, medicine-ball throw, modified pull-up, 20-meter run and 4-meter shuttle-run. The results indicate that the performance in all tests were higher among boys and increased directly with age group. These two factors (sex and age) were the main predictors of physical fitness amongst the investigated variables. Type of school influenced only the performance in the medicine-ball throw, being higher for students from private schools. Students from urban zone achieved a better performance in all tests, except in 
the modified pull-up, in which there was no difference between geographic regions. The development of researches with the same approach in other Brazilian regions will allow evaluating the consistency of these findings and understanding possible differences.

UNITERMS: Motor activity; Exercise; Athletic performance; Child; Adolescent.

\section{Referências}

AMERICAN ALLIANCE FOR HEALTH, PHYSICAL EDUCATION, RECREATION AND DANCE (AAHPERD) . Youth fitness test manual. Washington: AAHPERD, 1976.

COMMITTEE FOR THE DEVELOPMENT OF SPORT. Handbook for the EUROFIT tests of physical fitness. Rome: Council of Europe, 1988.

DUMITH, S.C.; AZEVEDO, M.R.; ROMBALDI, A.J. Aptidão física relacionada à saúde de alunos do ensino fundamental do município de Rio Grande, RS, Brasil. Revista Brasileira de Medicina do Esporte, São Paulo, v. 14, n. 5, p.454-59, 2008. GLANER, M.F. Importância da aptidão física relacionada à saúde. Revista Brasileira de Cineantropometria e Desempenho Humano, Florianópolis, v.5, n.2, p.75-85, 2003.

Aptidão física relacionada à saúde de adolescentes rurais e urbanos em relação a critérios de referência. Revista

Brasileira de Educação Física e Esporte, São Paulo, v.19, n.1, p.13-24, 2005.

GUEDES, D.P. Implicações associadas ao acompanhamento do desempenho motor de crianças e adolescentes. Revista Brasileira de Educação Física e Esporte, São Paulo, v.21, p.37-60, 2007. Número especial.

GUEDES, D.P.; GUEDES, J.E.R.P. Esforços físicos nos programas de educaçäo física escolar. Revista Paulista de Educação Física, São Paulo, v.15, n.1, p.33-44, 2001.

Crescimento, composição corporal e desempenho motor em crianças e adolescentes. Sao Paulo: CLR Balieiro, 2002. GUEDES, D.P.; GUEDES, J.E.R.P.; BARBOSA, D.S.; OLIVEIRA, J.A. Atividade física habitual e aptidão física relacionada à saúde em adolescentes. Revista Brasileira de Ciência e Movimento, Taguatinga, v.10, n.1, p.13-21, 2002. HALLAL, P.C.; MATSUDO, S.M.; MATSUDO, V.K.; ARAUJO, T.L.; ANDRADE, D.R.; BERTOLDI, A.D. Physical activity in adults from two Brazilian areas: similarities and differences. Cadernos de Saúde Pública, Rio de Janeiro, v.21, n.2, p.573-80, 2005.

KVAAVIK, E.; KLEPP, K.I.; TELL, G.S.; MEYER, H.E.; BATTY, G.D. Physical fitness and physical activity at age 13 years as predictors of cardiovascular disease risk factors at ages 15, 25, 33, and 40 years: extended follow-up of the Oslo Youth Study. Pediatrics, Elk Grove Village, v.123, n.1, p.e80-6, 2009.

MALINA, R.M. Tracking of physical activity and physical fitness across the lifespan. Research Quartely for Exercise and Sport, Washington, v.67, n.3, p.S48-57, 1996. Supplement.

MARQUES, A.C.; GAYA, A.; SILVA, G.; TORRES, L. Projeto Esporte Rio Grande do Sul - PROESP-RS. Perfil, Porto Alegre, v.7, n.1, p.9-11, 2005.

MORROW JUNIOR, J.R.; FULTON, J.E.; BRENER, N.D.; KOHL 3RD, H.W. Prevalence and correlates of physical fitness testing in U.S. schools-2000. Research Quartely for Exercise and Sport, Washington, v.79, n.2, p.141-8, 2008. ORTEGA, F.B.; RUIZ, J.R.; CASTILLO, M.J.; SJOSTROM, M. Physical fitness in childhood and adolescence: a powerful marker of health. International Journal of Obesity, London, v.32, n.1, p.1-11, 2008.

RIZZO, N.S.; RUIZ, J.R.; HURTIG-WENNLOF, A.; ORTEGA, F.B.; SJOSTROM, M. Relationship of physical activity, fitness, and fatness with clustered metabolic risk in children and adolescents: the European youth heart study. Journal of Pediatrics, Cincinnati, v.150, n.4, p.388-94, 2007.

RODRIGUES, L.P.; BEZERRA, P.; SARAIVA, L. Influência do meio (urbano e rural) no padrão de aptidão física de rapazes de Viana do Castelo, Portugal. Revista Portuguesa de Ciências do Desporto, Porto, v.5, n.1, p.77-84, 2005.

SILVA, M.A.; RIVERA, I.R.; FERRAZ, M.R.; PINHEIRO, A.J.; ALVES, S.W.; MOURA, A.A.; CARVALHO, A.C. Prevalência de fatores de risco cardiovascular em crianças e adolescentes da rede de ensino da cidade de Maceió. Arquivos Brasileiros de Cardiologia, Rio de Janeiro, v.84, n.5, p.387-92, 2005.

TOMKINSON, G.R.; OLDS, T.S. Secular changes in aerobic fitness test performance of Australian children and adolescents. Medicine and Sport Science, Basel, v.50, p.168-82, 2007.

TOMKINSON, G.R.; OLDS, T.S.; KANG, S.J.; KIM, D.Y. Secular trends in the aerobic fitness test performance and body mass index of Korean children and adolescents (1968-2000). International Journal of Sports Medicine, Stuttgart, v.28, n.4, p.314-20, 2007. 
TWISK, J.W.; KEMPER, H.C.; VAN MECHELEN, W. Tracking of activity and fitness and the relationship with cardiovascular disease risk factors. Medicine and Science in Sports and Exercise, Madison, v.32, n.8, p.1455-61, 2000. WELK, G.J.; MARROW, J.R.J.; FALLS, H.B. Fitnessgram reference guide. Dallas: The Cooper Institute, 2002. WESTERSTAHL, M.; BARNEKOW-BERGKVIST, M.; HEDBERG, G.; JANSSON, E. Secular trends in body dimensions and physical fitness among adolescents in Sweden from 1974 to 1995. Scandinavian Journal of Medicine and Science in Sports, Stockholm, v.13, n.2, p.128-37, 2003.

\section{Agradecimentos}

Agradecemos a toda a equipe envolvida no PROESP-BR, em especial ao coordenador do projeto no Rio Grande do Sul, Prof. Adroaldo Gaya. Os autores também agradecem aos diretores e professores das escolas que fizeram parte do estudo, bem como aos alunos e aos seus pais/responsáveis pelo consentimento em participar da pesquisa. O primeiro autor do artigo agradece ao CNPq pelo apoio concedido por meio de bolsa de estudos no momento da redação deste manuscrito.

Samuel Carvalho Dumith R. 6, 163 - Bairro Jardim do Sol 96216-09o - Rio Grande - RS - BRASIL e-mail: scdumith@yahoo.com.br
Recebido para publicação: 31/03/2009

Revisado em: 11/08/2009

Aceito: 26/08/2009

14 • Rev. bras. Educ. Fís. Esporte, São Paulo, v.24, n.1, p.5-14, jan./mar. 2010 